\title{
Systems Engineering approach in support to the breeding blanket design
}

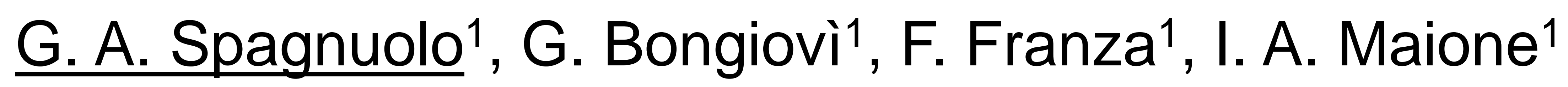

${ }^{1}$ Karlsruhe Institute of Technology (KIT), Institute for Neutron Physics and Reactor Technology (INR)

\section{Motivation}

$\square$ Capture, trace and maintain coherency between systems requirements

Manage large number of sub-system interdependencies

$\square$ Develop a holistic configuration to better understand the functional, spatial and physical integration aspects

\section{Logical/functional architecture using the} Systems Modelling Language (SySML)

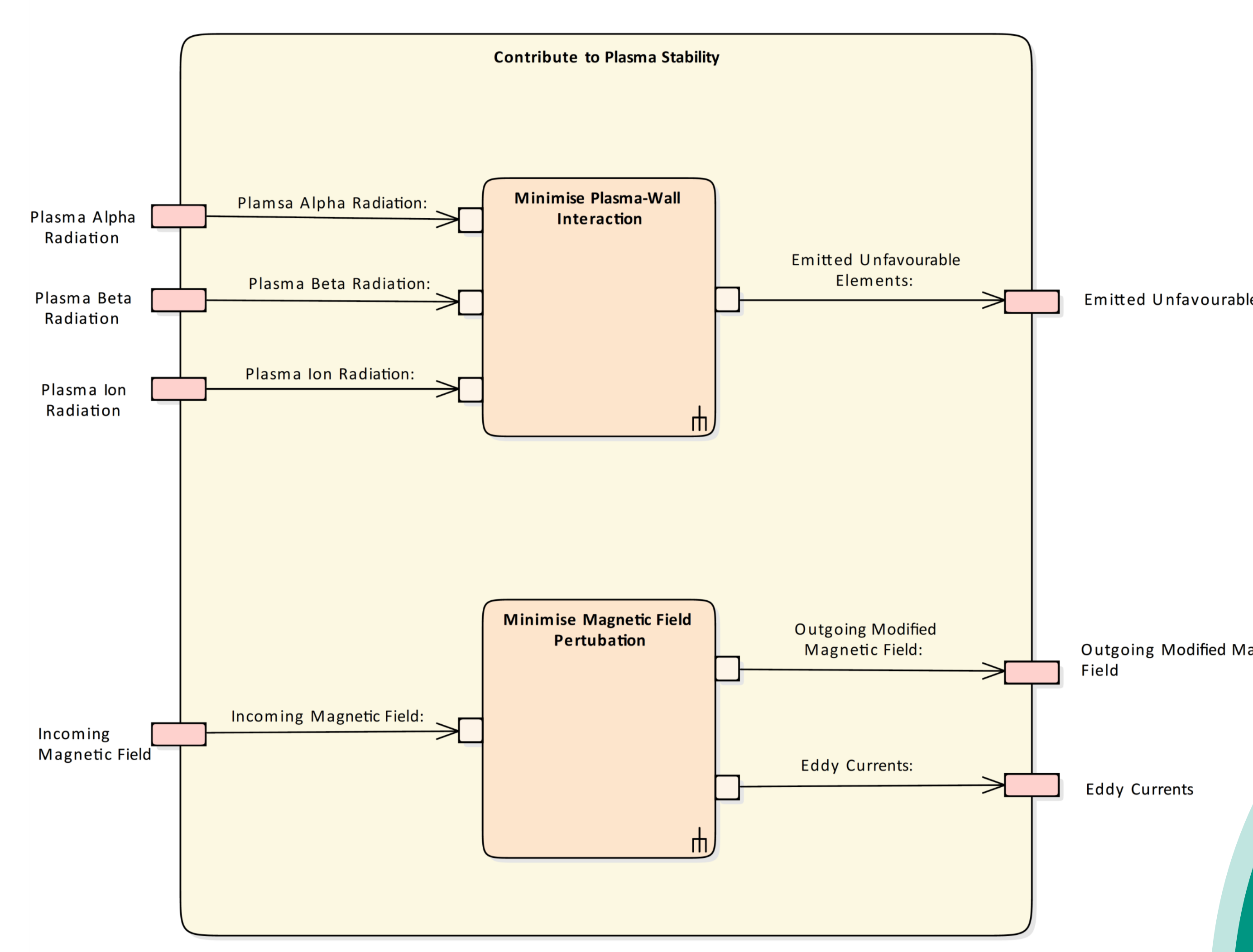

Functional, logical and physical levels defined in system architecture

$\square$ Solution-unrelated description of the design

$\square$ Illustration of system boundaries and model elements

\section{- Interface Management} Interface Identification

\section{Interface}

Definition (IDD)

Define Interface

Requirements (IR)

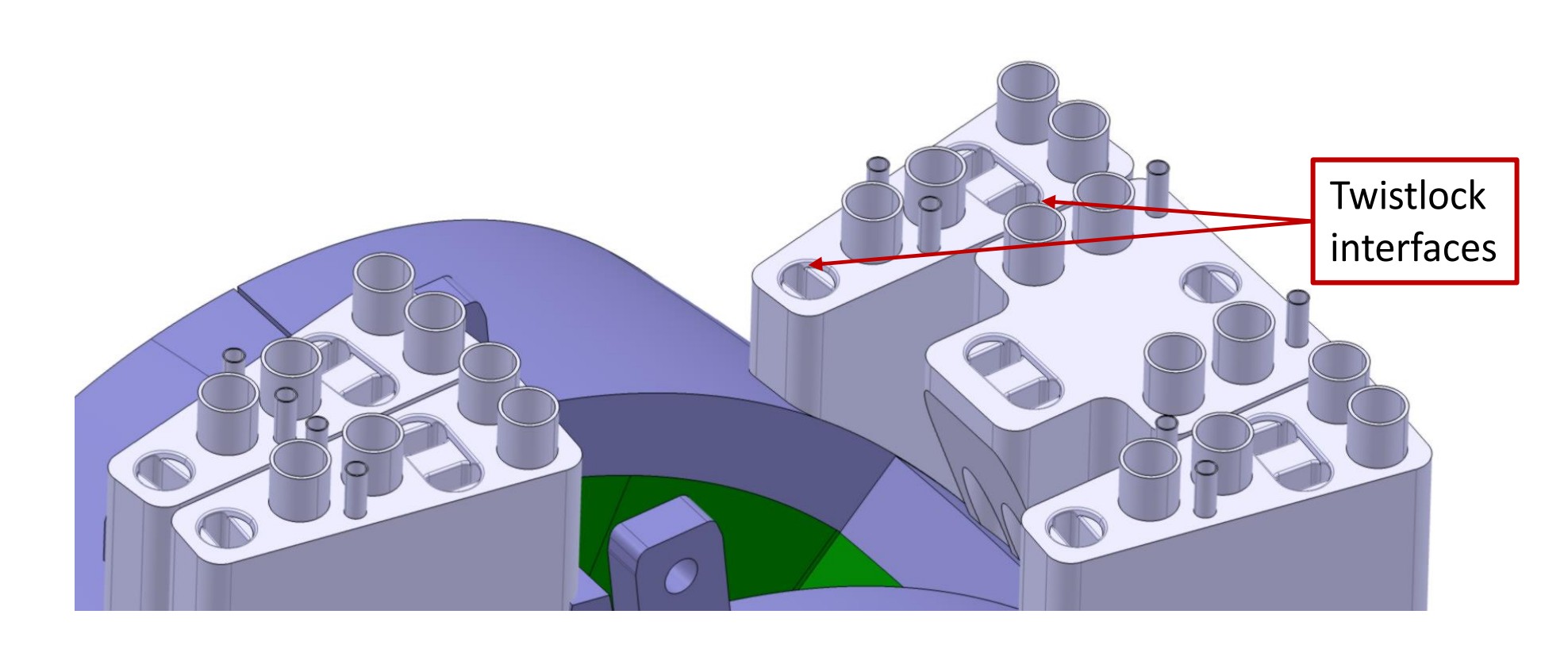

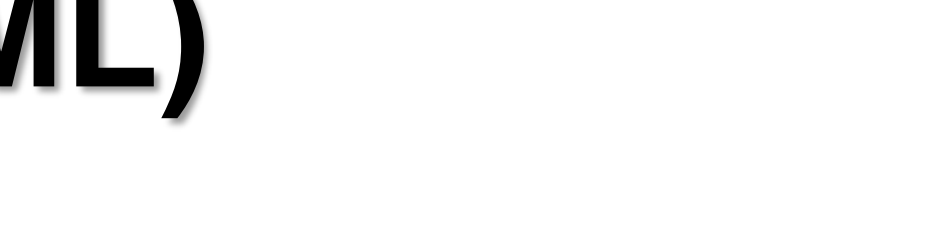

Interface Control (ICD)
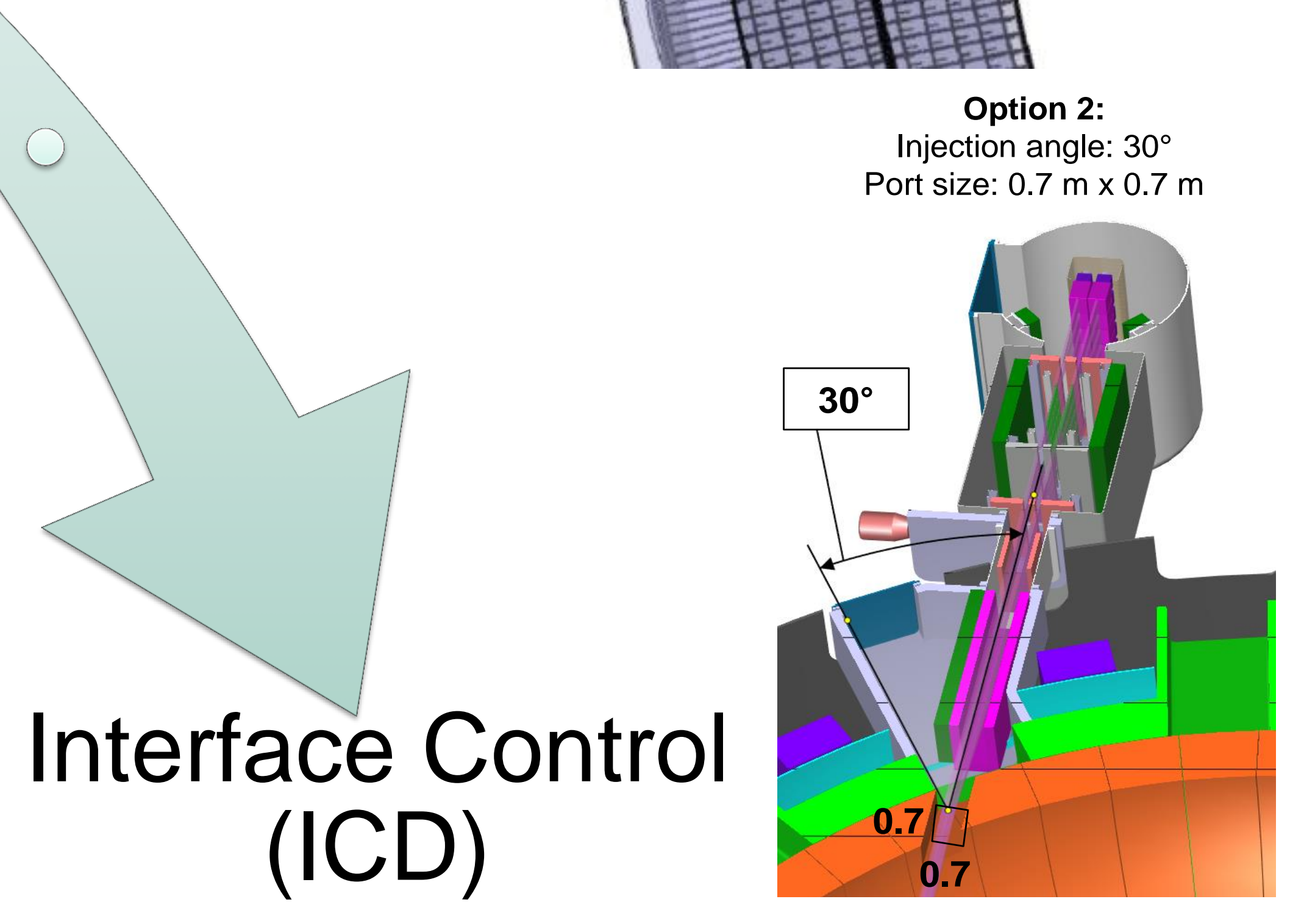

\section{System Requirement Document}

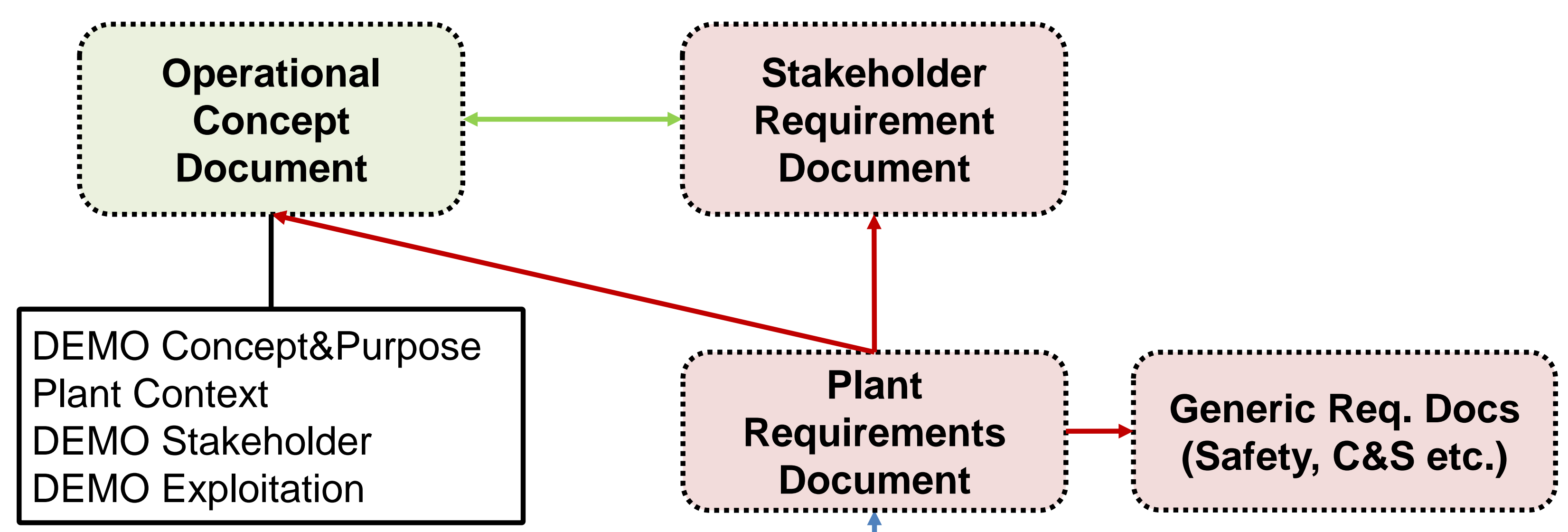 \\ System
Engineering \\ System
Engineering \\ in WPBB}

Plant Level $\overline{\text { System Level }}$

Functional flows (Control\&Objects) State machine cal architecture (plant/system levels) System interconno ant\&System cardinal design datpar) Plantesystem in Plant\&System performance and attributes

Plant Definition

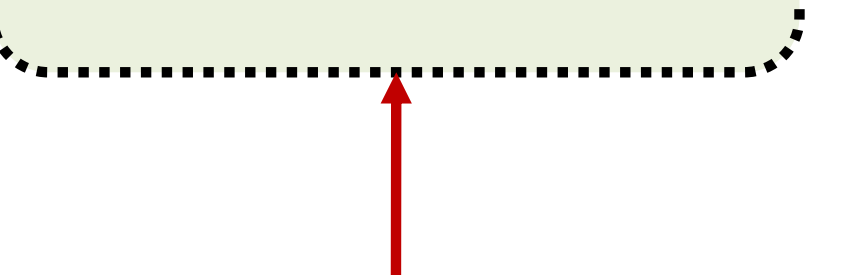

Breeding Blanket SRD

Breeding Blanket

Dom Definition Document

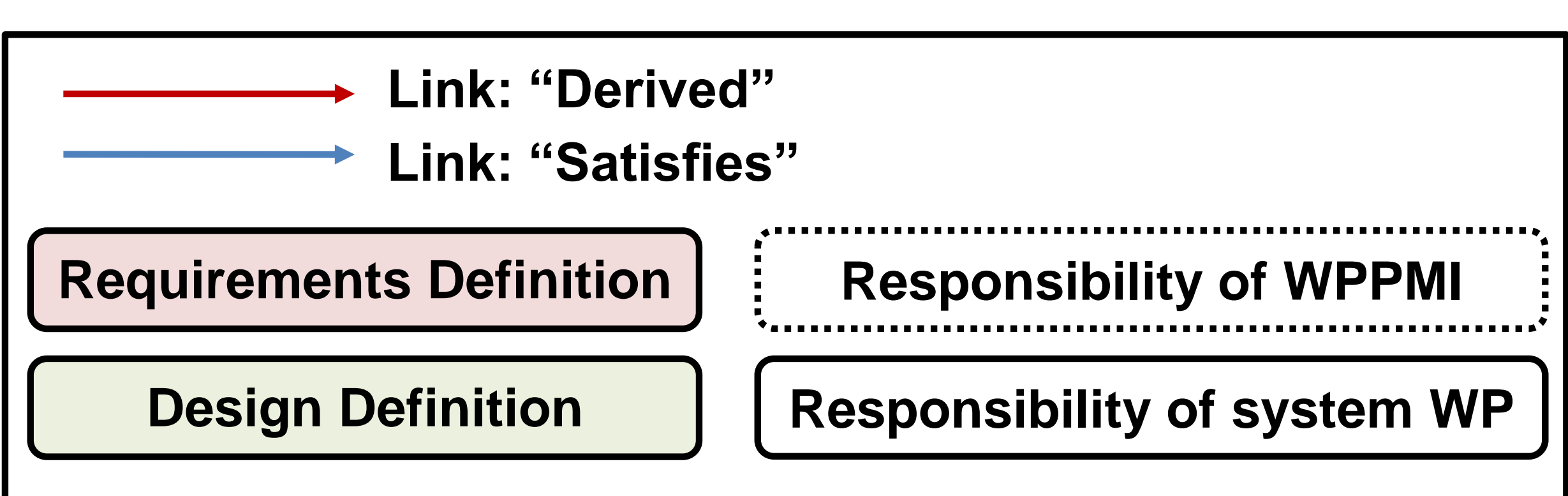

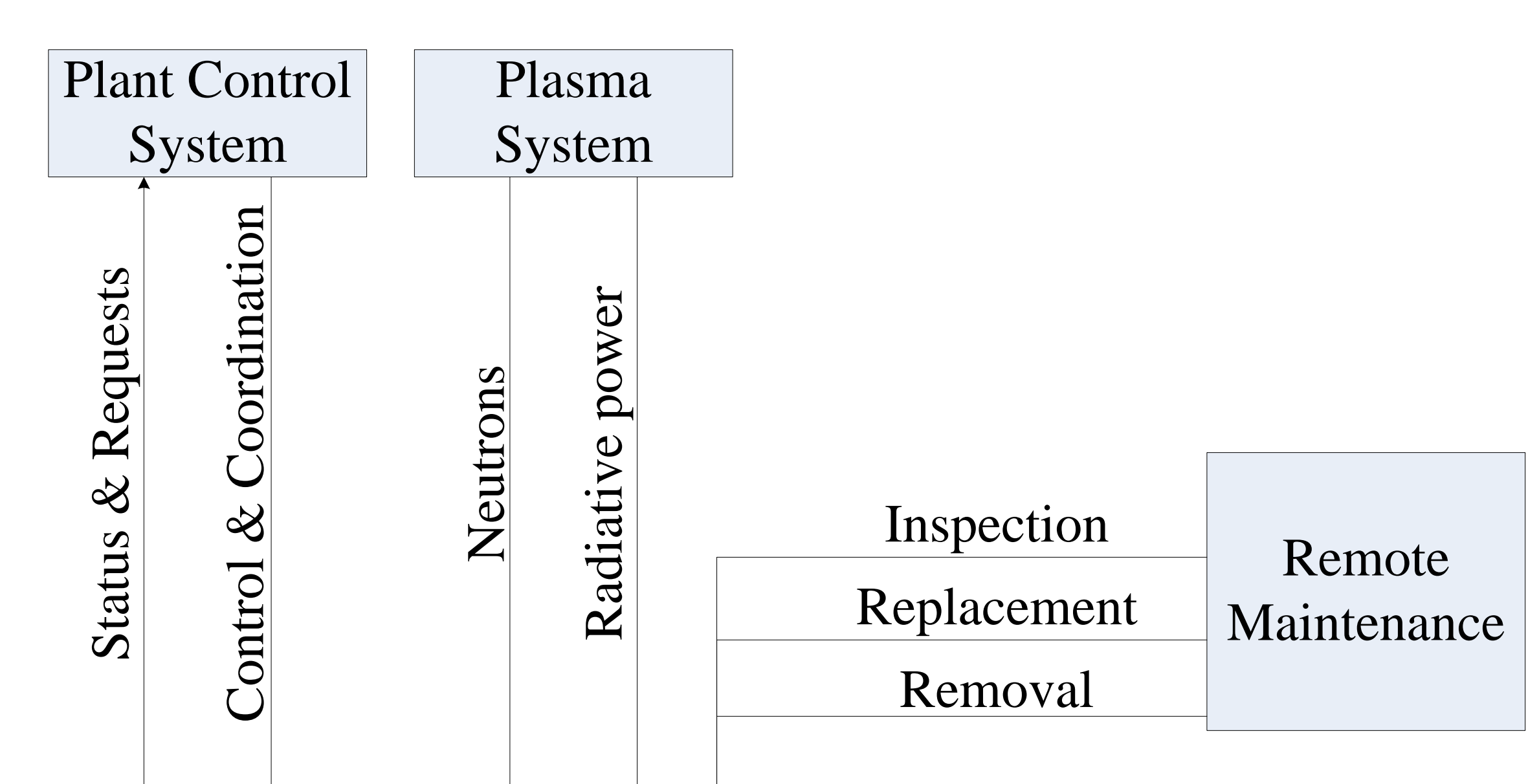

Thermal Power Primary Heat

Cooling Transfer

Coolant Pressure/

System

Coolant Inventory BB System *

Vacuum Vessel

\begin{tabular}{|l|l|}
\hline Upper Ports & Pipe Routing \\
\hline
\end{tabular}

Lower Ports Pipe Routing

Lower Ports Access

Attachments Physical Support

Nuclear Radiation Shielding

H\&CD Geometric Interface

Systems Geometric Integration

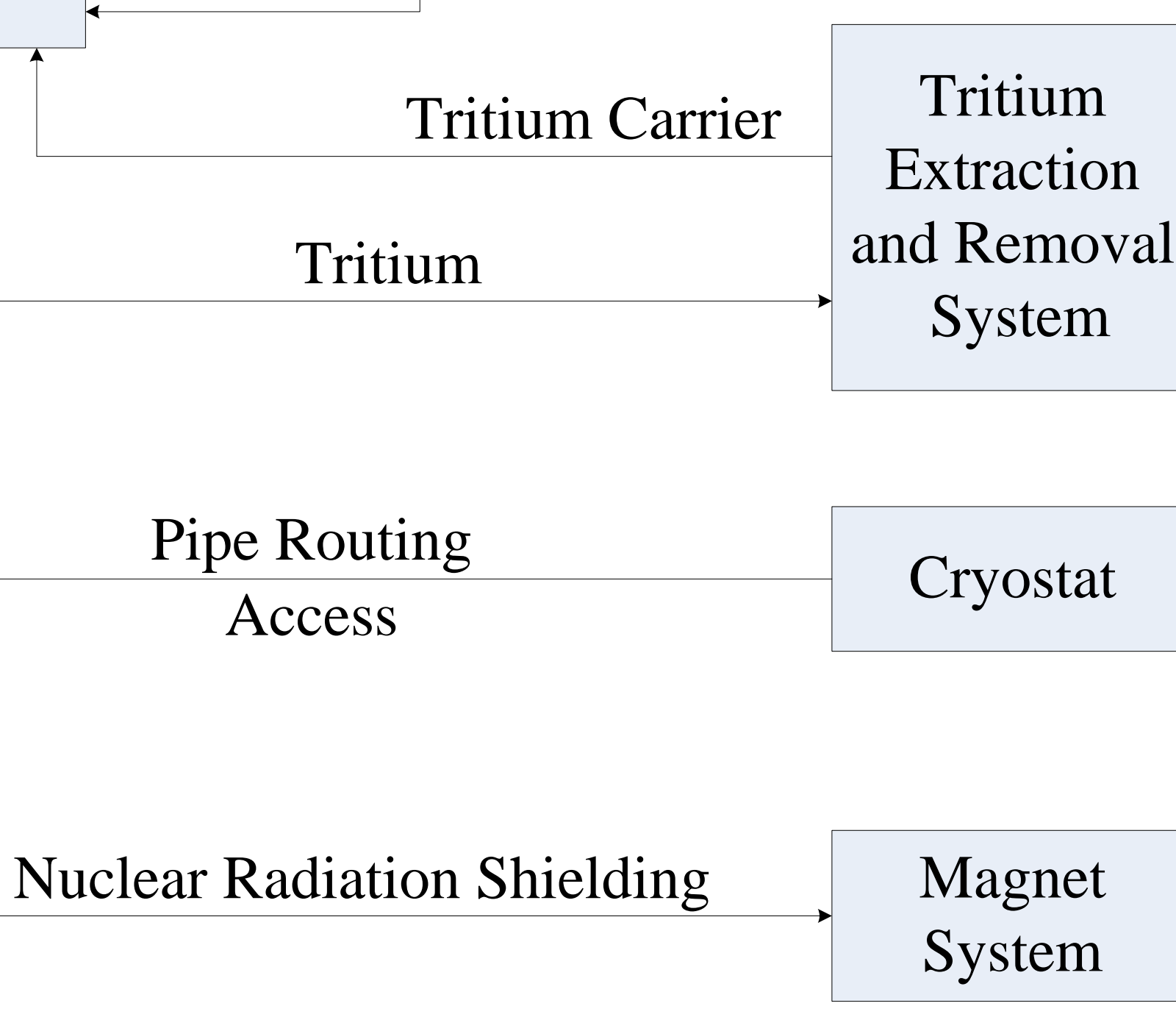

